\section{Interferon therapy for hepatitis}

\author{
from Arie J. Zuckerman
}

THE major importance of persistent infection with hepatitis $B$ virus and the carrier state can be considered first in terms of the potential dissemination of the infection to contacts by various parenteral (direct introduction into the tissues) and non-parenteral routes; and there are, at a conservative estimate, some 110 million carriers of hepatitis B surface antigen. Second, a significant proportion of infected patients and carriers have chronic active hepatitis, cirrhosis and probably primary liver cancer in certain areas of the world. Much has been written on this subject and considerable effort is being directed at passive and active immunisation. There is, however, no really effective treatment which affects the course of chronic infection with hepatitis $B$ virus, but somewhat unexpected and encouraging results have now been reported after systemic treatment with exogenous interferon, first by Greenberg and associates (New Engl. J. Med., 295,517 ; 1976) and more recently by Desmyter and colleagues (Lancet, ii, $645 ; 1976$ ).

Interferon has a well known broad spectrum antiviral action, and its lack of antigenicity in the homologous species and the absence of toxicity immediately provide a compelling appeal for its use. Indeed interferon has been investigated intensively since its discovery in 1957 by Isaacs and Lindenmann, and much effort has been devoted to its application in clinical practice for the treatment of several viral infections (Merigan et al. in Antiviral Mechanisms, Perspectives in Virology, 9 (edit. by Pollard, M.), 249; Academic, 1975). Interferon is probably not a single substance but rather it represents a class of cellular glycoproteins occurring as monomers or polymers of a basic unit with a molecular weight of $12,000-20,000$. It is synthesised by all vertebrates studied in response to viral infection and other intracellular parasites, polyanions, endotoxin and certain low molecular bis basic organic compounds. It is also produced as part of the cell-mediated immune response by lymphocytes.

There is experimental evidence that the viral inhibitory effect of interferon is at the level of translation of viral proteins; other data indicate that transcription of the viral genome is inhibited. But it now seems from other evidence that the viral inhibitory effect may be exerted not by interferon itself but by a cellular protein induced by interferon, and further that there is a range of proteins that can be considered as "interferons". Interferon also has an inhibitory effect on rapidly dividing cells including tumour cells, and its antitumour potential is under investigation. Interferon can be expected, therefore, to be more effective for prophylaxis than for the treatment of established virus diseases. However, in terms of disease process resulting from continuing viral replication and spread, interferon may be useful, for example by preventing the infection of fresh cells.

Greenberg et al. investigated the effect of exogenous human leukocyte interferon on persistent hepatitis B virus infection associated with chronic active hepatitis in four patients. The interferon was produced by $\mathrm{Dr}$ Kari Cantell by stimulation of human blood buffy coats with Sendai virus. The specific activity of the interferon was $5 \times 10^{5}$ units per $\mathrm{mg}$ protein. The four patients selected for the investigation were carriers of hepatitis B surface antigen for more than 6 months, and they had persistently abnormal liver function tests and histological changes in the liver consistent with chronic active hepatitis. In addition, three of the patients had high levels of circulating Dane particle markers including DNA polymerase, core antigen and Dane particle-associated DNA. Hepatitis $\mathbf{B} e$ antigen was present in these three patients, and the fourth patient had $e$ antibody (see Nature, 263, 374; 1976). The administration of interferon in a dose of between $6 \times 10^{3}$ and $17 \times 10^{4}$ units per $\mathrm{kg}$ per $\mathrm{d}$ was associated with a rapid and reproducible fall in DNA polymerase activity and the other Dane particle markers in the three patients on five separate occasions. This effect was transient when the interferon was given for up to $10 \mathrm{~d}$, but seemed to be more durable when the course of treatment lasted for a month or longer. Prolonged therapy was associated with the disappearance or diminution of the $e$ antigen in two of the patients. The effect of interferon on the surface antigen was more variable. Short term treatment had no effect on the titre of the surface antigen, but treatment for a month or longer was associated with a significant fall in titre in two patients.

Interferon thus seems to be exerting a suppressive effect on the production of Dane particles, the presumed infectious agent of hepatitis B. It is not known, however, at what stage of assembly of the Dane particle this effect occurs. Furthermore, interferon has also been shown to affect both humoral and cellular immunity, and Greenberg and associates point out that the suppression of the markers associated with hepatitis B virus may have been due to a direct antiviral action or mediated by the immune system or a combination of both. There is also the possibility that the active principle is not interferon but some other lymphokine including transfer factor (Nature, 258, 14; 1975) or other cell product which is present in the partially purified preparation collected from the human blood leukocytes. But this seems unlikely in view of the essentially similar results obtained by Desmyter et al. (loc. cit.) with interferon prepared in cultures of human diploid fibroblasts using the double-stranded RNA, poly(I) - $\operatorname{poly}(\mathrm{C})$, and superinduction. This preparation was given to two chimpanzee persistent carriers of hepatitis B surface antigen and one patient with antigen-positive chronic aggressive hepatitis. The treatment consisted of seven doses of $10^{7}$ international units of human interferon given on alternate days for 2 weeks. Interferon had no effect on the titre of the surface antigen of the two chimpanzee carriers but striking changes were observed in the hepatitis B core system. In the first chimpanzee, $15 \%$ of the nuclei of liver cells were found positive for core antigen by immunofluorescence. After treatment for 1 week, the number of positive nuclei and the intensity of staining decreased progressively during 5 weeks, and at that time $0.5 \%$ of the nuclei were positive. Then the number of positive nuclei increased rapidly reaching $30 \%$ after 1 month. The titre of circulating core antibody also increased. The second chimpanzee had no demonstrable core antigen in the liver cells at any time, but after treatment the high titre of serum core antibody fell sharply. In the patient, changes were seen in the core system. Intense core antigen staining was found in $30 \%$ of the liver cells before treatment. Immediately after treatment, $20 \%$ of the liver cell nuclei were positive at low intensity, and 5 weeks later only $3 \%$ were positive. The titre of core antibody in the serum remained unchanged.

These preliminary results obtained in short-term investigations in a few patients are promising and potentially most important, since interferon therapy may be useful in limiting the infectivity of carriers and it may perhaps eradicate chronic infection. The experimental hepatitis $\mathbf{B}$ vaccines currently under development are unlikely to achieve this in the foreseeable future. However, even the most ardent proponents of interferon and lymphokine therapy will urge carefully designed studies and only cautious optimism, for much remains to be learnt and accomplished before this form of treatment can be applied successfully to the many important clinical problems so clearly outlined by Merigan and his colleagues. 